\title{
Psychologische Aspekte des Systemumbruchs in den neuen Ländern nach der Wende: Einführung
}

\author{
Gisela Trommsdorff
}

\section{Ausgangsfragen zu psychologischen Folgen des Systemumbruchs}

Die staatlich vollzogene Vereinigung beider deutscher Staaten nach 40 Jahren eigener unterschiedlicher Entwicklung und dem Zusammenbruch des Systems der ehemaligen DDR hat mit dem vollständigen Systemwechsel in den neuen Ländern zu dramatischen Umbrüchen geführt. Es sind grundlegende Transformationsprozesse entstanden, die mit großem Tempo gleichzeitig auf Makro- und Mikroebene im politischen, wirtschaftlichen und sozialen Bereich zu gravierenden Veränderungen führen und massive Herausforderungen für die Menschen bedeuten. Die Vereinigung - von vielen gewünscht und gleichzeitig nicht erwartet - stellt nunmehr mit dem völligen Systemumbruch in den neuen Bundesländern erhebliche Anforderungen an die Menschen vor allem in Ostdeutschland. Die transformationsbedingten Rückwirkungen auf die alten Bundesländer stellen auch die Menschen dort vor neue Aufgaben, die jedoch hier nur in zweiter Linie berücksichtigt werden können. Die Herausforderungen in Ostdeutschland bedeuten für die Menschen dort erhebliche Belastungen aber auch besondere Chancen, die verhaltenswirksam sind und daher ihrerseits wiederum auf den TransformationsprozeB zurückwirken.

Zunächst wurde die Wende mit Euphorie umjubelt. Heute wird bereits die Frage nach einer doppelten Desillusionierung gestellt - zunächst in bezug auf den "Realsozialismus" und nunmehr in bezug auf die "soziale Marktwirtschaft" (vgl. Reilig, 1993). Gegenwärtig zeigen sich immer deutlicher Schwierigkeiten, über die wirtschaftliche und politische Vereinigung hinaus auch eine kulturelle und psychologische Integration zwischen Ost- und Westdeutschland zu erreichen. Heute ist die Rede von einigungsbedingten Krisen der politischen Akzeptanz, der Beschäftigung, der gesellschaftlichen Orientierung, der individuellen Verantwortung. Auch wenn diese Krisen kein direkter Effekt der Vereinigung sind - diese Probleme haben ihre eigentlichen Wurzeln in anderen Bedingungen - so sind sie doch durch die Transformationsprozesse verschärft worden.

Die gegenwärtigen Schwierigkeiten weisen darauf hin, daß ein vollständiger Systemwechsel offenbar nicht garantiert, da $B$ er die gewünschte Funktionsfähigkeit hat. Auch garantiert dies nicht, wie wir inzwischen erfahren haben, daß damit die "Vereinigung" bald abgeschlossen ist. Formal sind in den neuen Ländern gleiche politische Institutionen, Rechtsnormen, Regelungen, Verwaltungsvorschriften und Organisationsstrukturen in Wirtschaft und öffentlichem Leben übernommen worden. Abgesehen davon, daß viele Regelungen auch in den alten Ländern eigentlich änderungsbedürftig sind, ist fraglich, ob diese in gleicher Weise wie in der alten Bundesrepublik von den Menschen in Ostdeutschland verstanden, akzeptiert und ausgeführt, also in ihr Handeln integriert werden. Es kann nicht wirklich erwartet werden, daß mit einem formalen Systemwechsel die eigene politische Vergangenheit ausgelöscht und deren 
soziale und psychologische Folgen annulliert werden, und die Menschen automatisch die mit dem neuen System verbundenen Ziele und Kompetenzanforderungen übernehmen. Mit dem Institutionenwechsel können keineswegs gleichzeitig auch die mit dem damaligen System verbundenen Einstellungen und Verhaltensgewohnheiten einfach ausgetauscht werden. Nach dem Systemwechsel müssen die Menschen zunächst einmal Anpassungs- und Lernprozesse durchleben, die individuell sehr unterschiedlich und belastend sein können.

Die Umbrüche beschränken sich nicht auf die Änderung der Strukturen in Wirtschaft, Politik, Verwaltung und Bildungssystem. Bei diesen Transformationsprozessen geht es nicht nur darum, daß politische, administrative und legale Strukturen verändert werden; es sind schließlich Menschen, die in diesen Strukturen handeln, die diese Regeln ausführen und im Rahmen dieser Regeln handeln. Das Handeln erfolgt aufgrund bestimmter vorhandener Kompetenzen und Ziele, die mit diesen Strukturen mehr oder weniger gut im Einklang stehen. Schon daher sind die psychologischen Bedingungsfaktoren und Prozesse, die in der Transformation wirksam sind, relevant.

Wie die Prozesse in Institutionen und sozialen Gruppen verlaufen, hängt ganz wesentlich davon ab, welche psychologischen Bedingungen bei den Menschen bestehen, die in diesen Institutionen und sozialen Gruppen handeln. Je nachdem welche Ziele, Erwartungen, Wünsche und Fähigkeiten der Einzelne hat und in welchem sozialen und ökonomischen Kontext er eingebunden ist, wirkt sich dies auf die Wahrnehmung und die Verarbeitung geänderter Sachverhalte und Regeln verschieden aus.

Die individuellen Reaktionen auf die Vereinigung und auf die damit verbundenen Transformationsprozesse können als Anpassungsleistungen verstanden werden, die vom Individuum aus betrachtet - mehr oder weniger gelungen sind. Wie sich dies umgekehrt auf den Transformationsprozeß auswirkt, bleibt dabei zunächst offen. Es muß jedoch angenommen werden, daß gesellschaftliche Bedingungen und individuelles Handeln in wechselseitiger Beziehung zueinander stehen, und daß je nach der individuellen Verarbeitung auch die Transformationsprozesse in Tempo und Richtung beeinfluBt werden. Dabei handelt der Einzelne nicht isoliert, sondern eingebettet in soziale Gruppen. Der Transformationsprozeß beeinflußt also das Erleben und Verhalten des Einzelnen; andererseits wird sein Verlauf durch individuelles Handeln in verschiedenen sozio-ökonomischen und politischen Bereichen beeinfluBt, z.B. durch die Berufswahl und -tätigkeit, die Art der pólitischen Partizipation, durch Konsum- oder Bildungsverhalten. Darum können die individuellen Chancen und Risiken auch $\mathrm{zu}$ sozialen, politischen und wirtschaftlichen Chancen und Risiken werden. Daher genügt es nicht, sich nur auf die Strukturänderungen im Transformationsprozeß zu konzentrieren; vielmehr ist es erforderlich, auch die psychologischen Handlungsbedingungen und -folgen zu erfassen. Dazu will der vorliegende Band einen Beitrag leisten.

\section{Grenzen und Aufgaben des Bandes}

In diesem Band werden einige psychologische Ansätze aufgegriffen, die für die Beschreibung psychologischer Bedingungen und Folgen bei den Transformationsprozessen relevant sind. Die hier aufgenommenen empirischen Untersuchungen beruhen auf entwicklungs-, sozial- und organisationspsychologischen Ansätzen. Weitere Gemein- 
samkeiten der verschiedenen Beiträge des vorliegenden Bandes bestehen in einer "handlungstheoretischen" Grundposition - das Individuum als aktiven Organismus zu sehen, dessen Handeln in dem sozialen Kontext eingebunden ist - sowie darin, individuelle Orientierungen im Kontext des Systemumbruchs zu untersuchen. Dabei werden Muster individueller Orientierungen deutlich, die aus handlungstheoretischer Sicht besonders aufschlußreich sind, um die individuelle Verarbeitung von Transformationsprozessen zu analysieren. Gleichzeitig stellen sie Bindeglieder zwischen Prozessen sozio-politischen Wandels und individuellem Handeln dar.

Eine weitere Gemeinsamkeit der hier vorliegenden Beiträge ist, daß diese alle als vergleichende Studien konzipiert sind. Es werden Daten zum Vergleich von Stichproben aus den neuen und alten Bundesländern oder zum Vergleich von Stichproben, die vor und nach der Wende untersucht wurden, diskutiert. Wenn kein direkter systematischer Vergleich vorgenommen wurde, wird auf empirische Befunde vergleichbarer Studien zurückgegriffen. Aus naheliegenden Gründen sind Vergleiche zwischen Ostund Westberlin, die unmittelbar nach der Wende begonnen wurden, hier überrepräsentiert, obwohl Ost- und Westberlin besondere Kontexte darstellen, die keineswegs in dieser Weise für die neuen und die alten Bundesländer zutreffen müssen.

Diese Vergleiche zwischen Ost- und Westdeutschen sind auch aus anderen Gründen kritisch zu betrachten. Ähnlichkeiten können nicht schlicht im Sinne modemisierungstheoretischer Konvergenzthesen als Annäherung von Werten und Handlungszielen gedeutet und mit den im Westen typischen Mustern von Individualisierung und postmodernen Werthaltungen gleichgesetzt werden. Vielmehr ist zu fragen, ob anscheinend gleiche Phänomene (z.B. Werthaltungen) tatsächlich für Ost- und Westdeutsche das gleiche bedeuten, bzw. auch wie nachgewiesene Unterschiede zu interpretieren sind. Dazu sind eigentlich weiterreichende Untersuchungen erforderlich, die nicht nur singuläre Werthaltungen, Ziele etc., sondern auch deren Beziehungen untereinander im jeweiligen individuellen Orientierungssystem vergleichen, und vor allem darüber hinaus die Verhaltensrelevanz dieser Orientierungen im jeweiligen sozio-ökonomischen Kontext prüfen. Solche Fragen nach der Bedeutung von Ähnlichkeiten und Unterschieden bei Vergleichen zwischen Ost und West oder vor und nach der Wende berühren Probleme der funktionalen Äquivalenz der Daten (vgl. Trommsdorff, 1989; und in diesem Band); dies legt einen kritischen Umgang mit den Befunden, d.h. ihrer Bedeutung und Generalisierbarkeit, nahe.

Es ist natürlich hier nicht möglich, aufgrund der Vergleiche von Erhebungen kurz vor und kurz nach der Wende bereits Schlüsse über den Transformationsprozeß (oder auch die Wirkung des DDR-Systems) zu ziehen. Die Beiträge dieses Bandes lassen allenfalls Hypothesen für präzisere Fragestellungen zu. Auch darf man aufgrund von Daten zu individuellen Orientierungen und Erwartungen zu einem bestimmten Erhebungszeitpunkt nicht annehmen, daß diese ohne weiteres Vorhersagen für zukünftiges Verhalten der Befragten und den Verlauf der Transformationsprozesse erlauben. Solche Vorhersagen können die Beiträge hier nicht leisten; dazu sind empirische Studien zur Stabilität und Veränderung dieser psychologischen Merkmale im Rahmen veränderter Kontextbedingungen über die Zeit hinweg im Längsschnitt durchzuführen. Einige Studien dieses Bandes behandeln zwar entwicklungsspezifische Veränderungen in für die Transformation relevanten Bereichen. Die meisten der hier vorliegenden Studien beschreiben jedoch nur die zu einem bestimmten Zeitpunkt gegebenen psychologischen Phänomene und erhellen damit als "Momentaufnahmen" Ausschnitte aus dem 
Transformationsprozeß, die unter Berücksichtigung des Kontextes sozio-ökonomischer und politischer Gegebenheiten zu interpretieren sind.

Vorsicht ist auch bei der Generalisierung der Befunde geboten. Die meisten dieser Beiträge beziehen sich auf relativ kleine und meist nicht auf bevölkerungsrepräsentative Stichproben. Daten solcher Stichproben dienen dem in der Psychologie üblichen Ziel der Beschreibung individueller Besonderheiten und spezifischer Zusammenhangsmuster sowie der Generierung von Hypothesen für weiterführende Studien. Den kritischen Leser sollten bei der Lektüre dieses Bandes solche Überlegungen begleiten, um den Beitrag dieser Einzelstudien angemessen einordnen und den Verlauf der Transformationsprozesse besser verstehen zu können.

Im Rahmen dieses Bandes kann nicht das ganze Spektrum psychologischer Voraussetzungen, Begleiterscheinungen und Folgen der Transformation erfaßt und ein umfassendes Bild vom Denken, Erleben und Verhalten der ostdeutschen Bevölkerung gekennzeichnet werden, auch wenn anderweitig Versuche vorliegen, die griffig die "Mentalität" der Ostdeutschen entschlüsseln wollen (vgl. Maaz, 1991). In diesem Band werden keine aus einer umfassenden Theorie (die empirisch prüfbar wäre) abgeleiteten und alle Bereiche abdeckenden Analysen von Transformationsprozessen oder gar Prognosen geliefert. Vielmehr gehen wir sehr viel vorsichtiger vor. Hier werden empirische Daten zu relevanten psychologischen Phänomenen von Transformationsprozessen berichtet, die zwar teilweise mehrdeutig zu interpretieren, aber gut aufeinander zu beziehen sind, und die deutliche Muster individueller Orientierungen skizzieren.

Diese empirischen Daten gewähren Einblicke in psychologische Aspekte von Transformationsprozessen und in das Handeln der an diesem Prozeß beteiligten Menschen. Damit soll eine differenziertere Sichtweise über bestehende Zusammenhänge und eine Präzisierung psychologischer Aspekte der Transformationsprozesse erfolgen, um einen Beitrag zur Aufklärung der Folgen eines herausragenden historischen Ereignisses zu leisten. Bei systematischer Weiterführung einiger der hier aufgenommenen empirischen Untersuchungen ließen sich allerdings vermutlich brauchbare Prognosen und Anhaltspunkte für politische und wirtschaftliche Entscheidungen und Programme ableiten.

\section{Aufbau und Inhalt des Bandes}

Der Band ist in drei Teile gegliedert. Im ersten Teil werden einige theoretische Ansätze und Konzepte aus der Entwicklungs-, Sozial- und Persönlichkeitspsychologie vorgestellt, die erlauben, die besonderen psychologischen Vorgänge bei den Transformationsprozessen genauer zu analysieren. Der zweite Teil enthält empirische Einzelstudien, die relevante psychologische Phänomene im TransformationsprozeB wie Werthaltungen, Ziele, Kontroll- und Zukunftsorientierung sowie Aspekte sozialer Interaktion beschreiben. Da die Transformationsprozesse Menschen jeden Alters erfassen und lebensaltersspezifisch verschieden sind, wurden hier aus einer Lebensspannenperspektive die besonderen Bedingungen und Auswirkungen für Kinder, Jugendliche und Erwachsene behandelt. Im dritten Teil werden Einstellungen und Verhaltensweisen im Kontext des Arbeitsmarktes und des Berufes untersucht. In diesem Kontext bestehen besondere Anforderungen, die u.a. bei Berufsentscheidungen, bei der Perso- 
nalauswahl, in der Zusammenarbeit am Arbeitsplatz, bei Führungs- und Organisationsfragen sowie bei Arbeitslosigkeit wirksam werden.

\subsection{Theoretische Ansätze und Konzepte}

Im ersten Teil werden Transformationsprozesse und ihre Wirkungen aus persönlichkeits-, entwicklungs- und sozialpsychologischer Perspektive behandelt. Trommsdorff diskutiert zunächst, worin die psychologischen Probleme bei der gegenwärtigen Umbruchsituation bestehen und wieso dies Aspekte von Wechselwirkungen zwischen Individuum und Umwelt sind. Sie stellt dann einige Konzepte und theoretische Ansätze vor, mit denen sich die Folgen der Umbrüche für den Einzelnen genauer analysieren lassen, und die Schnittstellen zwischen dem sozio-ökonomischen Wandel einerseits und den psychologischen Prozessen andererseits herausarbeiten. Damit wird auch versucht, die hier vorliegenden Beiträge im Rahmen übergreifender theoretischer Fragestellungen einzuordnen. Es werden allgemeine psychologische Phänomene bei der Konfrontation mit neuen oder bei Verlust gewohnter Handlungsoptionen sowie individuelle Unterschiede im Umgang mit den Transformationsprozessen, z.B. aufgrund kohortenspezifisch verschiedener Sozialisation und in verschiedenen Kontexten diskutiert. Verarbeitungsprozesse werden am Beispiel von Identität, Kontroll- und $\mathrm{Zu}$ kunftsorientierung (u.a. unter Bezug zu Beiträgen in diesem Band) behandelt. Befunde zu Ähnlichkeiten und Unterschieden von psychologischen Merkmalen bei Ost- und Westdeutschen werden u.a. im Hinblick auf methodische Probleme bei solchen Vergleichsstudien erörtert.

Im nächsten Kapitel stellen Schmidt und Heckhausen zentrale entwicklungspsychologische Theorien und Konzepte dar, die sich eignen, um Anpassungen im Transformationsprozeß aus einer Lebensspannenperspektive $\mathrm{zu}$ analysieren. Sie greifen dabei u.a. die einflußreichen Arbeiten von Glen Elder (1974) auf, um die Bedeutung von Entwicklungsalter und Kohorte bei der Bewältigung von sozio-ökonomischen Umbrüchen zu belegen. Weiter präzisieren sie Anpassungsprobleme im Rahmen des Modells der selektiven Optimierung von Baltes und Baltes (1989; 1990) (vgl. auch Heckhausen, in diesem Band). Nach diesem Modell wird vermutet, daß Menschen mit zunehmendem Alter versuchen, die bereits investierten Aufwendungen durch besondere kompensatorische Bemühungen in ihrem Gesamtnutzen zu erhalten. Durch die Transformationsprozesse können jedoch die gesellschaftlichen Stützen für Selektion und Kompensation zusammenbrechen und bisherige selektive Investitionen in die eigene Entwicklung verloren gehen. Ähnlich wie bei "kritischen Lebensereignissen" erfordert dies unerwartete Anpassungsleistungen.

Im folgenden Beitrag diskutiert Montada psychologische Probleme der Vereinigung aus der Sicht der Gerechtigkeitsforschung. Wenn man davon ausgeht, daß sich in der ALLBUS-Basisumfrage von 1991 gezeigt hat, daß die Deutschen in den alten und neuen Bundesländern die eigene Lage in bezug auf Verteilungsgerechtigkeit völlig unterschiedlich einschätzen - Westdeutsche sahen sich zu diesem Zeitpunkt als überpriviligiert und Ostdeutsche als benachteiligt (vgl. Noll, 1992) - so wäre eigentlich zu erwarten, daß Westdeutsche zu Umverteilungen, Investitionen und Transferleistungen in den Osten bereit sind. Dies ist jedoch keineswegs selbstverständlich. Die Gründe dafür und die möglichen Folgen für die Transformationsprozesse diskutiert Montada. 
Er konfrontiert aktuelle sozio-politische Probleme der Einigung mit gerechtigkeitspsychologischen Theorieansätzen. Montada geht davon aus, daß zentrale Probleme der Transformationsprozesse durch subjektiv wahrgenommene Ungleichheiten (u.a. auf dem Arbeitsmarkt und in bezug auf den Lebensstandard) und die Selbst- und Fremdwahrnehmung der Opfer-Position entstehen. Die dadurch begründeten Forderungen nach Umverteilungen können sich je nach subjektiv wahrgenommener Ungerechtigkeit (die wiederum von bestimmten Deutungsvoreingenommenheiten abhängt) verschärfen. Aus der Forschung zur Verteilungsgerechtigkeit und zum Hilfehandeln werden darüber hinaus Fragen und Hypothesen zu den Chancen und Risiken des weiteren Verlaufs der Transformationsprozesse abgeleitet, die wiederum für einige der folgenden Beiträge über psychologische Teilaspekte der Transformation relevant sind (z.B. Schmitt und Janetzko; Bergmann, in diesem Band). Montada entwickelt nicht nur Grundlagen für weiterführende theoretische Forschungen, sondern legt auch praktische Implikationen für politische Planung nahe.

\subsection{Entwicklungs- und sozialpsychologische Aspekte der Transformation in der Lebensspanne}

In diesem zweiten Teil werden Transformationsprozesse unter der Perspektive der Lebensspanne diskutiert und Probleme im Kindes-, Jugend- und Erwachsenenalter behandelt. Dabei wird davon ausgegangen, daß in jedem Entwicklungsalter eigene spezifische Anforderungen bestehen, die zusätzlich zu den Anforderungen der Transformation vom Einzelnen zu bewältigen sind. Diese können Chancen aber auch Risiken bedeuten. Es werden hier grundlegende Werthaltungen und Ziele, Zukunfts- und Kontrollerwartungen erörtert, die auf spezifische Anpassungsprobleme im Transformationsprozeß hinweisen. Darüber hinaus werden psychologische Aspekte sozialer Interaktion behandelt, die in den meisten Studien zur Transformation vernachlässigt wurden (kindlicher Spracherwerb und interpersonale Wahrnehmung der Menschen beider Teile Deutschlands). Dies sind Aspekte, durch die die institutionalisierte Einigung auf der Ebene individueller Einstellungen und interpersonaler Beziehungen erheblich gestört oder gefördert werden kann.

\subsubsection{Werthaltungen und Erziehungsziele}

Krettenauer, Edelstein, Dettenborn, Doil, Grundmann und Schmieschek untersuchen die Bedeutung von Transformationsprozessen für einen zentralen Bereich der kognitivsozialen Entwicklung: die soziale und moralische Orientierung (u.a. politische Werthaltungen, soziale Einstellungen, moralische Urteilskompetenz). Die Autoren gehen von der Frage aus, in welcher Weise die sozio-moralische Entwicklung von makro- und mikrosozialen sowie von individuellen Bedingungen beeinflußt wird. Um Einflüsse der Transformationsprozesse von ohnehin stattfindenden intraindividuellen Entwicklungsprozessen zu trennen, bzw. um Kohorten- und Alterseffekte zu isolieren, wurden hier Quer- und Längsschnittstudien beim Vergleich von Jugendlichen aus Ostund Westberlin durchgeführt. Die Autoren nehmen an, daß 15-Jährige aufgrund ihrer noch nicht stabilisierten Identitätsentwicklung als eine Risikogruppe zu sehen sind, die 
in besonderem Maße sensibel für transformationsbedingte Belastungen ist. Tatsächlich unterstützen die Befunde die Annahme, daß Jugendliche, die die Transformationsprozesse in der frühen Adoleszenz erleben und vermutlich noch nicht über die nötigen sozial-kognitiven Ressourcen zur Bewältigung verfügen, eher mit anomischen, aggressiven und materialistischen Tendenzen reagieren. $\mathrm{Ob}$ solche Orientierungsprobleme tatsächlich für diese Altersgruppe typisch sind, ob diese Phänomene vorübergehend auftreten oder (für diese Altersgruppe) dauernde Folgen haben, wird weiter zu prüfen sein.

Der Beitrag von Schnabel, Baumert und Roeder befaßt sich mit dem Vergleich von Werthaltungen, zum einen auf der Grundlage von Sekundäranalysen bevölkerungsrepräsentativer Befragungen (ALLBUS) sowie zum anderen aufgrund ergänzender eigener Befragungen an größeren Stichproben. Dabei werden über Ost-West-Vergleiche hinaus vor allem innerhalb der beiden Regionen Vergleiche nach Alter und Bildung durchgeführt, um mögliche generationenspezifische und sozio-strukturelle Effekte zu erfassen, die differentielle Zusammenhänge mit den Transformationsprozessen aufklären sollen. Es zeigt sich u.a., daß in den neuen und alten Bundesländem soziale Unterschiede in Werthaltungen über die Schulbildung reproduziert werden. Deutliche Alterseffekte belegen, daß die ostdeutsche jüngere Generation eher als die ältere postmaterialistische Werte vertritt und darin der westlichen Bevölkerung ähnlich ist. Die größten Unterschiede beim Ost-West-Vergleich postmaterialistischer Werthaltungen treten bei den 25 - bis 40-Jährigen auf. Jugendliche und Erwachsene sind sich in den neuen Bundesländern weniger ähnlich als in den alten Bundesländern. Die Befunde deuten für die junge Generation auf eine Konvergenz von Werthaltungen zwischen beiden Teilen Deutschlands hin (vgl. auch Jülisch et al., in diesem Band). Zu fragen ist nach diesen Befunden, ob Intergenerationskonflikte in den neuen Bundesländern zu erwarten sind, die eine neue Qualität von nicht nur auf die Familie beschränkten Spannungen auslösen könnten.

Ahnert, Kratzig, Meischner und Schmidt stellen eine Untersuchung über mütterliche Erziehungsziele für Kleinkinder vor. Die Autoren zeigen, daß keine signifikanten Unterschiede in Erziehungszielen im Vergleich der beiden deutschen Stichproben (Osnabrück, Ostberlin) vor der Wende und auch keine Unterschiede im Vergleich der ostberliner Mütter vor und nach der Wende bestehen. Hingegen unterscheiden sich Mütter aus Moskau und Ostberlin. Demgegenüber zeigen Ergebnisse anderer Studien (Sturzbecher, 1993) erhebliche "Kultur"-Unterschiede zwischen ost- und westdeutschen Eltern in bezug auf Erziehungsziele kurz vor der Wende (z.B. besonders in bezug auf Anpassung und Hilfsbereitschaft). Auf der Grundlage der hier vorliegenden sehr kleinen Stichproben und ohne Daten zu Erziehungszielen aus den 60er und 70er Jahren verfügbar zu haben, läßt sich daher wohl nicht ohne weiteres darauf schließen, daß Werte und Erziehungsvorstellungen in Ost- und Westdeutschland trotz 40 Jahre unterschiedlicher Systemwirkungen gleich geblieben sind. Über diesen Vergleich hinaus werden hier ost- und westdeutsche mit russischen Müttern verglichen. Die Einbeziehung einer osteuropäischen Stichprobe, die ebenfalls von Transformationsprozessen erfaßt ist, erlaubt, über den deutsch-deutschen Vergleich hinausgehende Fragen zu sozialisationsrelevanten Bedingungen zu untersuchen. Dieses Vorgehen könnte bei entsprechender Stichprobengröße und Erfüllung methodischer Voraussetzungen des Kulturvergleichs allgemeine Prozesse von Transformationen aufzuklären helfen. 


\subsubsection{Kontroll-und Zukunfisorientierungen}

Oettingen und Little untersuchen in ihrem Beitrag die Frage, ob sich Grundschulkinder in einem für die weitere soziale und kognitive Entwicklung relevanten Bereich - in der Beurteilung leistungsbezogener Selbstwirksamkeit - unterscheiden, wenn sie in der ehemaligen DDR oder in der alten Bundesrepublik aufgewachsen sind. Die Autoren gehen von attribuierungstheoretischen Ansätzen aus, nach denen eine gewisse positive Selbstüberschätzung und Überschätzung eigener Kontrollmöglichkeiten förderlich für die Bewältigung von schwierigen Aufgaben ist. Die theoretisch abgeleitete Hypothese, daß bestimmte Einflüsse (u.a. "öffentliche" Selbsteinschätzung in der Schulklasse), wie sie im Schulsystem der ehemaligen DDR üblich waren, eine positive Überschätzung verhindern, wird im Vergleich ost- und westberliner Grundschüler getestet. Tatsächlich stellen die Autoren (trotz Ähnlichkeiten in Entwicklungstrends) fest, daß ost- im Vergleich zu westberliner Schüler weniger positive Überzeugungen über ihre Begabung und Anstrengungsfähigkeit haben und insgesamt stärker die Lehrerurteile übernehmen. Die Autoren erwarten besonders für leistungsschwache Schüler demotivierende Effekte solcher vergleichsweise pessimistischeren Selbstwirksamkeitsurteile. Dies müßte jedoch in entsprechend angelegten Längsschnittstudien unter Berücksichtigung des Unterrichtsstils im einzelnen geprüft werden, denn hier werden Fragen der Identität, Kontrollüberzeugung, Zukunftsorientierung und Leistungsbereitschaft tangiert, die als wichtige psychologische Voraussetzungen für erfolgreiche Anpassungsleistungen im Transformationsproze $B$ angesehen werden können (vgl. Trommsdorff; Schnabel, Baumert und Roeder; Heckhausen; Jülisch, Sydow und Wagner, alle in diesem Band).

Heckhausen untersucht mögliche Unterschiede in Lebenszielen bei ost- und westberliner Erwachsenen. Sie geht in ihrem Beitrag davon aus, daß der Zerfall bisheriger gesellschaftlicher Strukturen die individuelle Regulation des Lebenslaufs beeinträchtigt und individuelle Ziele und Kontrollorientierungen beeinflußt. Gemäß dem Modell von Baltes und Baltes (1989) (vgl. Schmidt \& Heckhausen, in diesem Band) nimmt die Autorin an, daß die Menschen in den neuen Ländern versuchen, die Kontinuität ihres Lebenslaufes trotz veränderter soziostruktureller Bedingungen zu sichern, indem sie verstärkt "primäre" Kontrolle ausüben und u.a. ihre Ziele hartnäckiger verfolgen. Die Stichprobengröße der befragten ost- und westberliner Erwachsenen erlaubt, Untergruppen nach Geschlecht, Alter und Berufseinfluß zu bilden, um Varianzen in der ostund westdeutschen Population aufzuklären (dies versuchen auch Jülisch, Sydow und Wagner; Schnabel, Baumert und Roeder, in diesem Band). Die geringere Überzeugung von eigenem Einfluß und eigenem Erfolg läßt sich im Zusammenhang mit den Befunden von Oettingen und Little (in diesem Band) sehen, die weniger positive (leistungsbezogene) Selbstwirksamkeitsurteile von ost- im Vergleich zu westberliner Grundschülern nachweisen. Allerdings sind Stichproben, Verfahren und Urteilsgegenstand verschieden. Die von Heckhausen nachgewiesene kürzere Zeitperspektive ostberliner Erwachsener entspricht Befunden von Jülisch et al. (in diesem Band) für ostund westberliner Jugendliche. Allerdings ist noch zu klären, was die kürzere Zeitperspektive subjektiv bedeutet: Spricht dies für eine größere Dringlichkeit und Ungeduld beim Anstreben von Zielen? Bedeutet dies eine größere Sorge, daß befürchtete Ereignisse früher eintreten? Werden realistische Ziele gesetzt, die in naher Zukunft erreichbar sind? 
Der Frage nach der Art von Zukunftserwartungen bei ost- und westberliner Jugendlichen gehen Jülisch, Sydow und Wagner in ihrem Beitrag nach. Hier werden zukunftsbezogene Anpassungsprobleme im Rahmen subjektiver Lebenstheorien von Jugendlichen untersucht. Die Autoren gehen von der Annahme aus, daß die durch den Systemwandel bedingten Änderungen identitätswirksam sind und die Zukunftsvorstellungen von Jugendlichen beeinflussen. Die Autoren begnügen sich nicht mit einem globalen Vergleich ost- und westberliner Schüler; sie untersuchen eine ausreichend große Stichprobe, um Schüler verschiedener Schultypen zu vergleichen. Außerdem nutzen sie den methodischen Vorzug operanter Verfahren und lassen ihre Probanden selbst Hoffnungen und Befürchtungen nennen, die diese in bezug auf vorgegebene, theoretisch relevante Zukunftsaspekte (Ursachenzuschreibung etc.) bearbeiten. Ostim Vergleich zu westberliner Schüler haben eine weniger ausgedehnte Zeitperspektive in bezug auf ihre Hoffnungen und Befürchtungen, und sie nennen mehr politisch relevante Zukunftserwartungen. Inhaltlich stimmen jedoch die Zukunftsvorstellungen weitgehend überein und beziehen sich auf Grundbedürfnisse. Auffällig sind $\mathrm{Ge}-$ schlechtsunterschiede und Unterschiede zwischen Real- und Gesamtschülem und Gymnasiasten in beiden Teilen Berlins. Diese Befunde, die im übrigen vorsichtig als "Momentaufnahmen" gedeutet werden, unterstreichen die Notwendigkeit, den "interkulturellen" Ost-West-Vergleich durch "intrakulturelle" Vergleiche zu ergänzen und der "intrakulturellen" Varianz durch Einbeziehung relevanter Variablen (bzw. Untergruppen) gerecht zu werden, um die Vergleiche zu differenzieren.

Auch die Studie von Oettingen trägt zum Vergleich von Ost- und Westberlinern in bezug auf optimistische und pessimistische Einstellungen einen interessanten Aspekt bei. Die Autorin verwendet nicht wie sonst üblich Befragungen. Sie führte vor der Wende in Ost- und Westberlin Inhaltsanalysen von Medienberichten in bezug auf pessimistische Attribuierungen durch; weiter beobachtete sie vor und nach der Wende die Mimik von Kneipenbesuchern in Ost- und Westberlin in bezug auf depressive Verstimmtheit. Während sich vor der Wende deutliche Unterschiede zeigten - Depressivitätsmerkmale waren in Ostberlin ausgeprägter - sind diese nach der Wende nicht mehr erkennbar. Stichprobenbeschreibungen für die beobachteten Personen liegen (wie bei Feldbeobachtungen meist üblich) nicht vor. Auch wenn kritisch zu prüfen ist, welchen Indikatorwert diese Verfahren für emotionale Stimmungen haben, ist $z u$ hoffen, daß diese Arbeit Studien zur Wirkung von Transformationsprozessen auf emotionale Stimmungen im Alltag und deren Bedeutung für Anpassungsprozesse anregt.

\subsubsection{Aspekte sozialer Interaktion}

In dem Beitrag von Friedrich und Lehwald wird die Sprachentwicklung von Vorschulkindern vor und nach der Wende untersucht, um den möglichen Einfluß veränderter Sozialisationsbedingungen zu prüfen. Dies erfolgt zu einem für die Sprachentwicklung sensiblen Entwicklungszeitpunkt. Die institutionalisierte Vorschulerziehung, die in der ehemaligen DDR einem einheitlichen Erziehungsplan folgte und mehr als zwei Drittel aller Klein- und Vorschulkinder erfaßte (vor der Wende besuchten Kleinkinder bereits vor dem ersten Lebensjahr zu mehr als 70\% eine Krippe), hat sich seit dem Beitritt vollständig geändert. Kinder sind längere Zeiten in der Familie; Erzieherinnen müssen nicht mehr einem Programm folgen, das großen Wert auf die Spra- 
chentwicklung (und dort auf die Beherrschung der Grammatik) legt. Die mit der Wende verbundenen theoretisch relevanten Einflußgrößen lassen sich bei diesem Vergleich also relativ genau beschreiben. Das erweist sich auch als notwendig, da die Ergebnisse eine Reihe von Fragen zu möglichen Ursachen der nachgewiesenen Unterschiede in Quantität und Qualität der Sprachproduktion bei Dreijährigen vor und nach der Wende offen lassen. Es bieten sich verschiedene Erklärungen der Befunde an, die in Fortsetzungsstudien bei entsprechender Kontrolle von Einflußvariablen empirisch geprüft werden könnten. Hier wäre durchaus ein Ansatz gegeben, spezifische, theoretisch relevante Aspekte der Wende - Verunsicherung von Erziehern; häufiger Fernsehkonsum bei Kindern; veränderte Kommunikationsstile in der Familie - für ein quasi-natürliches Experiment zu nutzen.

Während sich die bisherigen Beiträge vor allem mit psychologischen Problemen der Transformationsprozesse bei Ostdeutschen befassen, geht es in dem Beitrag von Schmitt und Janetzko um spezifische Aspekte sozialer Interaktion zwischen Ost- und Westdeutschen, und zwar in bezug auf die Selbst- und Fremdwahrnehmung. Ist die formale Einigung auch im Bewußtsein der Deutschen verankert, und ist die politische Vereinigung mit einer mentalen Einigung verbunden? $\mathrm{Zu}$ diesen Fragen geben Daten dieser Studie eine Teilantwort. Hier werden Voraussetzungen für ein "Zusammenwachsen" beider Teile Deutschlands angesprochen. Dabei ist nicht nur nach der Anpassung von Ostdeutschen sondern auch der von Westdeutschen zu fragen und zwar in bezug auf die Wirkung für die innere Integration, die sich u.a. in der sozialen Interaktion manifestiert (vgl. auch Wottawa, in diesem Band). Wie groB die Kluft zwischen Ost- und Westdeutschen ist, ließe sich u.a. darin sehen, wie ähnlich oder unterschiedlich sich Ost- und Westdeutsche erleben. Die Autoren zeigen, daß die Stereotype, die jeweils jede Bevölkerungsgruppe über die andere Gruppe hat, sehr ähnlich sind. Das würde eine Integrationshyothese stützen, wenn sich die Inhalte dieser Stereotype ähnelten. Ost- und Westdeutsche nehmen sich jedoch keineswegs als ähnlich, sondern als sehr verschieden wahr. Dabei bewerten sie sich selbst jeweils positiver. Diese Studie ist (trotz methodischer Probleme bei der Datenanalyse in einem differenzierten Untersuchungsdesign) auch darum so interessant, weil sie die Veränderung von Hypothesen und Perspektiven der Autoren im Prozeß ihrer Arbeit zeigt. Die Autoren sind zunächst von der Annahme ausgegangen, daß sich ost- und westdeutsche Erwachsene in bezug auf Attribuierungen von Verantwortlichkeit (und Kontrollierbarkeit von Schadensfällen) unterscheiden. Diese Hypothese wurde jedoch durch die empirischen Daten widerlegt. Daraufhin fragten die Autoren, inwieweit ihre eigene Hypothese eine "westliche" sei, und wie sich Personen aus den alten Bundesländern selbst und wie sie Personen aus den neuen Bundesländern in bezug auf Verantwortlichkeit (für bestimmte Schadensfälle) beurteilen.

Ost- und Westdeutsche zeigen jeweils typische Diskriminierungstendenzen der jeweils anderen Gruppe, wie sie aus der Vorurteilsforschung bekannt sind, wenn das Bedürfnis nach Schaffung sozialer Identität (vgl. Tajfel, 1982) tangiert ist. Beide, Ostund Westdeutsche stereotypisieren im Sinne der günstigeren Beurteilung der "Eigengruppe" und der weniger günstigen Beurteilung der "Fremdgruppe". Ost- und Westdeutsche nehmen sich jeweils als "Fremde" wahr. Wie gravierend und integrationshemmend dies ist, müBte durch entsprechende Vergleiche verschiedener kulturellregionaler Gruppen innerhalb Ost- und Westdeutschlands aufgeklärt werden. Weiter stellt sich für Folgestudien die Frage, welche Konsequenzen solche Verzerrungen in 
der Eigen- und Fremdwahrnehmung für den Umgang miteinander haben, z.B. am Arbeitsplatz und im Alltag.

\subsection{Einstellungen und Verhalten im wirtschaftlichen Umbruch}

Im dritten Teil werden psychologische Aspekte des wirtschaftlichen Umbruchs behandelt. Die Übernahme des Wirtschafts-, Rechts- und Verwaltungssystems, von Technologie und Personal aus Westdeutschland bewirkt allein noch keine grundlegende Änderung der ostdeutschen Wirtschaft. Abgesehen von massiven strukturellen und wirtschaftlichen Problemen tragen psychologische Faktoren dazu bei, wie der Systemwechsel wirksam wird. Offenbar haben sich bestimmte Bereiche wirtschaftsrelevanten Handelns bei den Menschen aus den neuen Ländern bisher kaum verändert und scheinen den Anforderungen des neuen marktwirtschaftlichen Systems nicht zu entsprechen.

Der Beitrag von Rappensperger, Rosenstiel und Zwarg belegt für Bewerbungsgespräche deutliche Unterschiede in Präferenzen und Zielen ost- und westdeutscher Bewerber. An zahlreichen Beispielen aus Interviews illustrieren sie, daß Ostdeutsche im Vergleich zu Westdeutschen persönliche Aspekte und soziale Sicherheit bei der Stellensuche bevorzugen und Techniken der Selbstdarstellung und Werthaltungen der sozialen Marktwirtschaft eher ablehnen. $\mathrm{Zu}$ fragen ist, wie sich diese Einstellungen auswirken: ob dies eine relative Benachteiligung ostdeutscher Bewerber bedingt; wie dies das Verhalten am Arbeitsplatz beeinflußt; und ob die ablehnende Haltung gegenüber der sozialen Marktwirtschaft aufgrund eigener (durch eigenes Verhalten evtl. auch provozierter) negativer Erfahrungen weiter verstärkt wird. Zu prüfen wäre in Folgestudien, ob die hier nachgewiesenen Einstellungsunterschiede eine Integration der Ostdeutschen in das System der sozialen Marktwirtschaft erschweren.

Nach den Ergebnissen von Frese und Hilligloh zeigen Ost- im Vergleich zu Westdeutschen gegenwärtig geringere arbeitsbezogene, aber mehr soziale Eigeninitiative am Arbeitsplatz (z.B. Veranstaltungen organisieren). Als Datenbasis werden repräsentative Befragungen (Interviews) in zwei Städten verwendet. Wenn man jedoch berücksichtigt, daß Eigeninitiative in der Arbeit mit hohem Handlungsspielraum am Arbeitsplatz und mit der Überzeugung, Handlungen effektiv ausführen $z u$ können (Selbstwirksamkeit) zusammenhängt, so ist anzunehmen, daß die Einführung entsprechender Bedingungen am Arbeitsplatz die Bereitschaft zur Eigeninitiative fördern müßte. Zu bedenken ist jedoch, daß Eigeninitiative allein für wirtschaftliches Handeln sicher nicht ausreicht - sie könnte ja auch als Aktionismus ins Leere gehen und muB zumindest von VerantwortungsbewuBtsein, Problemlösefähigkeit und anderen Kompetenzen ergänzt werden. Singuläre Merkmale zu fördern, kann disfunktional sein, je nachdem welche Beziehung zu anderen Merkmalen besteht. Vielmehr sind diese wie andere Persönlichkeitsmerkmale (Selbständigkeit, Flexibilität) ein Ergebnis des Zusammenwirkens von individuellen und situativen Bedingungen. Dabei spielen vergangene Erfahrungen sowie aber auch die aktuellen situativen Bedingungen eine Rolle. Während allerdings Eigeninitiative und Selbständigkeit für manche Mitarbeiter einen hohen Anreizwert haben können, sind diese Merkmale für andere Mitarbeiter (z.B. je nach eingeschätzten eigenen Kompetenzen und Anforderungen der Situation) eher bedrohlich. Hier werden die Befunde zur Entwicklung von Selbstwirksamkeits- und 
Kontrollüberzeugungen (vgl. Oettingen \& Little; Heckhausen, in diesem Band) relevant. Auch wenn die Arbeitsplatzbedingungen objektiv gleich sein sollten, können sie für Ost- und Westdeutsche etwas Unterschiedliches bedeuten und damit zu verschiedenen Handlungstendenzen führen. Daher ist sicher wichtig, Arbeitsbedingungen zu schaffen, die nicht unbedingt formal gleich sind, die aber günstige Voraussetzungen von Mitarbeitern (wie z.B. die bei ost- im Vergleich zu westdeutschen Führungskräfte-Bewerbern höhere intrinsische Arbeitsmotivation) aufgreifen und zur Entfaltung bringen.

Schwierig wird dieser Versuch einer optimalen Passung von Mitarbeiterpersönlichkeit und Arbeitsplatzbedingung besonders durch die Einbindung der Mitarbeiter in soziale Interaktionen am Arbeitsplatz. Besonders für den Fall von Interaktionen zwischen ost- und westdeutschem Personal können für beide Seiten unerwünschte Probleme entstehen. Wottawa diskutiert daher am Beispiel von Interaktionszirkeln zwischen west- und ostdeutschen Führungskräften, wie die jeweils zwar unterschiedlichen, aber durchaus für die anstehenden Aufgaben jeweils funktionalen Persönlichkeitsmerkmale durch Mißverständnisse und Fehlwahrnehmungen die Kooperation behindern. Eine anfängliche Zurückhaltung von Ost-Mitarbeitem kann sich aufgrund der dadurch erhöhten Aktivität von West-Mitarbeitern, die ihrerseits unter Erfolgsdruck stehen, verstärken. Oder eine auf Machtdistanz beruhende formale Anpassung ostdeutscher Mitarbeiter macht bei gleichzeitiger Ablehnung des "Mächtigeren" eine funktionierende Kooperation wenig wahrscheinlich. Wenn gegenseitige negative Stereotype bestehen, die die eigene Identität gegenüber der anderen Gruppe positiv absichern sollen, ist die Kooperationsgrundlage ebenfalls sehr problematisch. Bei diesen Interaktionsprozessen dürfte die gegenseitige Wahmehmung verstärkend wirksam sein und müßte ähnlich, wie bei Schmitt und Janetzko (in diesem Band), eigens untersucht werden. Es bedarf besonderer Sorgfalt, wenn westdeutsches Führungspersonal ostdeutsche Mitarbeiter zu schulen, zu beurteilen oder einzustellen hat. Die erforderliche intensive Zusammenarbeit von Personen aus ganz unterschiedlichen Lebenskontexten stellt sowohl ost- als auch westdeutsches Personal vor neue schwierige Aufgaben. Daher ist Wottawa zuzustimmen, daB eine sachgerechte Betreuung und Schulung gerade auch von westdeutschen Führungskräften für ihre Tätigkeit in den neuen Ländern erforderlich ist.

Die wachsende Arbeitslosigkeit in Ostdeutschland ist wohl eines der schwierigsten Probleme des Transformationsprozesses. Die Befunde von Bergmann deuten darauf hin, daß gegenwärtig (bzw. zum Untersuchungszeitpunkt) noch eine hohe Bereitschaft zur Anpassung und Akzeptanz von wirtschaftlicher und persönlicher Schlechterstellung bei ostdeutschen Arbeitslosen besteht. Bergmann befragte Arbeitslose aus dem Raum Dresden. Da diese Region nicht zu den wirtschaftsschwächsten gehört, wäre eine Vergleichsanalyse mit einer größeren Stichprobe von Arbeitslosen aus einer besonders strukturschwachen Region sowie ein Vergleich mit westdeutschen Arbeitslosen sicher aufschlußreich. Nach den Befunden von Bergmann scheint es - anders als bei Studien zu westdeutschen Arbeitslosen - noch ein Potential von Geduld, "sekundärer" Kontrollüberzeugung, Frustrationstoleranz und sozialem Rückhalt bei ostdeutschen Arbeitslosen zu geben. Allerdings werden individuelle Differenzen bestehen, die hier nicht dargestellt sind (auch die Streuung der Antworten wird nicht berichtet). Die Anpassungsbereitschaft könnte jedoch verspielt werden, wenn die individuelle Zeitperspektive und Bereitschaft zum Aufschub von erhoffter Zielerfüllung 
überfordert werden, weil sich keine erfolgsversprechenden Handlungsoptionen zeigen. Dies legen inzwischen andere Studien nahe; z.B. weisen Kallabis, Liljeberg und Hesse (1992) in einer von der Kommission für die Erforschung des sozialen und politischen Wandels in den neuen Bundesländern e.V. (KSPW) geförderten Studie deutliche negative soziale und psychologische Folgen von Arbeitslosigkeit in Ostdeutschland nach.

\section{Zusammenfassung}

Ob (oder unter welchen Bedingungen) die Menschen aus beiden Teilen Deutschlands als Folge ihrer Sozialisation in ganz unterschiedlichen Systemen bzw. als Folge der unterschiedlichen gegenwärtigen Lebensumstände - unterschiedlich denken, fühlen und handeln und wie dies wiederum die Transformationsprozesse beeinflußt, muß über "Momentaufnahmen" hinaus wiederum empirisch geprüft werden. Weiter ist zu fragen, an welchen Stellen individuelle und soziale Anpassungen und Änderungen zur Lösung anstehender Probleme möglich sind. Der Systemwechsel allein bewirkt jedenfalls noch kein Zusammenwachsen. Ob und wie sich die Menschen der neuen Bundesländern in das neue System (u.a. in die freie Marktwirtschaft) integrieren, hängt wohl auch damit zusammen, wie sich die Menschen aus den alten Bundesländem auf die Transformationsprozesse einstellen. Der Transformations- und Einigungsprozeß wird über institutionelle Regelungen hinaus durch individuelle Anpassungen im Erleben und Verhalten und durch zwischenpersönlichen Austausch vermittelt. Bereits die jeweiligen subjektiven Vorstellungen über sich selbst und über die Menschen im jeweils anderen Teil Deutschlands sind Grundlagen für diesen Austausch. Bei der Begegnung der Menschen aus den alten und neuen Bundesländern bestehen gegenwärtig auf beiden Seiten erhebliche Voreingenommenheiten und Vorbehalte. Die psychologische Kluft scheint gewachsen zu sein.

Auch empirische Befunde über individuelle Orientierungen der Menschen in den neuen Bundesländern sind in Gefahr, nicht nur vom Forscher selbst, sondern auch von den Rezipienten als Bestätigung bestehender Vorurteile und Überzeugungen selektiv verwendet zu werden. Teilweise widersprüchliche Befunde in den Beiträgen dieses Bandes sowie die Darstellung ausgewählter spezifischer Aspekte psychologischer Faktoren im Transformationsprozeß mögen der Versuchung zu voreiligen Generalisierungen entgegenwirken und gleichzeitig zu gezielten Folgestudien ermuntern, die der Aufklärung der Transformationsphänomene dienen.

\section{Literatur}

Baltes, P.B. \& Baltes, M.M. (1989). Optimierung durch Selektion und Kompensation: Ein psychologisches Modell erfolgreichen Alterns, Zeitschrift für Pädagogik, 35, 85-105.

Baltes, P.B., \& Baltes, M.M (1990). Psychological perspectives on successful aging: The model of selective optimization with compensation. In P.B. Baltes \& M.M. Baltes (Eds.), Successful aging. Perspectives from the behavioral sciences (pp. 1-34). Cambridge: Cambridge University Press.

Elder, G. (1974). Children of the great depression. Social change in life experience. Chicago: University of Chicago Press. 
Kallabis, H., Liljeberg, H. \& Jesse, J. (1992). Zur Spezifik der Arbeitslosigkeit in den neuen Bundesländern - soziale und psychische Folgen - individuelle und soziale Săttigung. Kurzstudie Nr.5152. Unveröffentlichtes Manuskript. Halle: Kommission für die Erforschung des sozialen und politischen Wandels in den neuen Bundesländern e.V. (KSPW).

Maaz, H.-J. (1991). Das gestürtzte Volk oder die unglückliche Einheit. Berlin: Argon.

Noll, H.H. (1992). Zur Legitimität sozialer Ungleichheit in Deutschland: Subjektive Wahrnehmungen und Bewertungen. In P.Ph. Mohler \& W. Bandilla, W. (Hrsg.), Blickpunkt Gesellschaft 2. Einstellungen und Verhalten der Bundesbürger in Ost und West (S. 1-20). Opladen: Westdeutscher Verlag.

Reilig, R. (1993). TransformationsprozeB Ostdeutschlands - Entwicklungsstand - Konflikte - Perspektiven. In R. Reißig (Hrsg.), Rückweg in die Zukunft. Über den schwierigen Transformationsprozeß in Ostdeutschland. (S. 11-48). Frankfurt, New York: Campus.

Sturzbecher, D. (1993). Jugendliche in Brandenburg - Auf der Suche nach Orientierung. Vortrag zur Fachkonferenz "Entstehung von Fremdenfeindlichkeit - Die Verantwortung von Politik und Medien" der Friedrich-Ebert-Stiftung (22.03.1993). Potsdam.

Taifel, H. (1982). Social identity and intergroup relations. London: Cambridge University Press.

Trommsdorff, G. (1989). Sozialisation und Werthaltungen im Kulturvergleich. In G. Trommsdorff (Hrsg.), Sozialisation im Kulturvergleich (S. 97-121). Stuttgart: Enke. 